\title{
Internal Auditory Canal Diverticula among Pediatric Patients: Prevalence and Assessment for Hearing Loss and Anatomic Associations
}

\author{
(DP.M. Bunch, (D) M.E. Zapadka, (D) C.M. Lack, (DE.P. Kiell, (DD.J. Kirse, and (D).R. Sachs
}

\begin{abstract}
BACKGROUND AND PURPOSE: Internal auditory canal diverticula are focal lucencies along the anterior-inferior aspect of the internal auditory canal fundus. Studies in adults report conflicting data on the etiology and clinical relevance of this finding. We would expect a pediatric study to help elucidate the significance of internal auditory canal diverticula. The primary goals of this study were to determine the temporal bone CT prevalence of diverticula among pediatric patients and to assess possible hearing loss and anatomic associations.
\end{abstract}

MATERIALS AND METHODS: For this retrospective study including 283 pediatric temporal bone CTs, 4 neuroradiologists independently assessed for diverticula. Discrepancies were resolved by consensus. One neuroradiologist assessed for an enlarged vestibular aqueduct, labyrinthine dysplasia, cochlear cleft, and otospongiosis. Patient demographics, audiologic data, and pertinent clinical history were recorded. One-way analysis of variance and the Fisher exact test were used to assess possible associations between diverticula and specific patient characteristics.

RESULTS: Diverticula were observed in 42/283 patients (14.8\%) and were more commonly bilateral. There was no significant association with age, sex, hearing loss, enlarged vestibular aqueduct, labyrinthine dysplasia, or cochlear cleft. A statistically significant association was observed with otospongiosis $(P=.013)$, though only 1 study patient had this disease.

CONCLUSIONS: Internal auditory canal diverticula are a common finding on pediatric temporal bone CT. In the absence of clinical or imaging evidence for otospongiosis, diverticula likely fall within the range of a normal anatomic variation. Familiarity with these findings may prevent neuroradiologists from recommending unnecessary additional testing in pediatric patients with isolated internal auditory canal diverticula.

ABBREVIATION: IAC = internal auditory canal

nternal auditory canal (IAC) diverticula are focal, well-demarcated osseous lucencies along the anterior-inferior aspect of the IAC fundus. ${ }^{1,2}$ This finding has also been referred to as "cavitary otospongiosis"3-5 and IAC "cupping."

Previous radiologic studies of IAC diverticula report conflicting data on the underlying etiology and clinical relevance of this finding. More specifically, one study of temporal bone CT examinations obtained in a cohort of 47 patients with otospongiosis

Received April 3, 2020; accepted after revision May 22.

From the Departments of Radiology (P.M.B., M.E.Z., C.M.L., J.R.S.), Otolaryngology, Head and Neck Surgery (E.P.K., D.J.K.), and Pediatrics (D.J.K.), Wake Forest School of Medicine, Winston Salem, North Carolina.

Previously presented as an electronic scientific poster at: Annual Meeting of the American Society of Head and Neck Radiology, October 2-6, 2019; Scottsdale, Arizona.

Please address correspondence to P.M. Bunch, MD, Department of Radiology, Wake Forest School of Medicine, Medical Center Blvd, Winston Salem, NC 27157; e-mail: paul.m.bunch@gmail.com; @pbunchmd

http://dx.doi.org/10.3174/ajnr.A6691 identified IAC diverticula in $>30 \%$ of otospongiotic temporal bones and characterized the finding as representing cavitary otospongiosis. ${ }^{5}$ However, temporal bone CT studies performed in larger cohorts report IAC diverticula in the absence of otospongiosis (ie, isolated IAC diverticula), with a prevalence of $\sim 5 \% .{ }^{1,8}$ One of these studies reported an independent association between isolated IAC diverticula and sensorineural hearing loss, ${ }^{8}$ and the other concluded that isolated IAC diverticula represent a normal anatomic variant not associated with hearing loss. ${ }^{1}$

These previous radiologic studies of IAC diverticula have been performed predominantly ${ }^{1}$ or exclusively ${ }^{5,8,9}$ in adults with no peer-reviewed published literature currently available on the prevalence of IAC diverticula among children. Given the rarity of otospongiosis among children and adolescents, ${ }^{10}$ we expect that a study of IAC diverticula among pediatric patients would help elucidate whether isolated IAC diverticula represent normal anatomic variants or acquired pathology. We hypothesize that if 
isolated IAC diverticula represent a normal variant, the pediatric prevalence should be comparable with the adult prevalence. Conversely, if isolated IAC diverticula are a manifestation of acquired pathology (eg, otospongiosis or other), we would expect the prevalence among children to be less than that in adults. The primary goals of this study were the following: 1) to determine the prevalence of IAC diverticula among pediatric patients on temporal bone CT, 2) to assess possible associations between IAC diverticula and hearing loss, and 3) to assess possible associations between IAC diverticula and other temporal bone CT findings of interest.

Most previous radiologic studies of IAC diverticula were performed with a single neuroradiologist reader. ${ }^{1,8,9}$ Moreover, there is variability among these studies with respect to what is defined as constituting an IAC diverticulum. One previous study included 2 neuroradiologist readers, though interrater reliability was not reported in this study. ${ }^{5}$ In our clinical practice, we have encountered uncertainty as to whether a mild contour irregularity of the IAC is sufficient to be classified as an IAC diverticulum. If IAC diverticula represent a clinically relevant finding, uncertainty and variability in radiologic diagnosis would be problematic. Thus, a secondary goal of this study was to assess interobserver variability with respect to what practicing neuroradiologists identify as IAC diverticula based on descriptions in the existing literature.

\section{MATERIALS AND METHODS \\ Subjects}

For this retrospective, Health Insurance Portability and Accountability Act-compliant, institutional review boardapproved study, a local institutional radiology data base (mPower; Nuance Healthcare) was queried for patients satisfying the following inclusion criteria: 1) age younger than 18 years, and 2) temporal bone CT examination performed between December 2015 and February 2019. Examinations were excluded if a temporal bone CT had already been included in the study cohort for the same patient. Additionally, unilateral temporal bones were excluded if the diagnostic assessment of the IAC was precluded by severe artifacts or if no IAC was present.

\section{Medical Record Review}

Patient age, patient sex, and the presence or absence of a clinical diagnosis of otospongiosis were recorded. When available, audiogram data were also reviewed and used to classify hearing as normal, sensorineural hearing loss, conductive hearing loss, or mixed hearing loss.

\section{Image Acquisition}

All temporal bone CT examinations were performed within the health system of our institution on multidetector row CT systems. Given the retrospective nature of this study, there was variability with respect to CT scanners used to acquire images as well as specific CT acquisition parameters. However, most temporal bone CT examinations were acquired on a Discovery $750 \mathrm{HD}$ (GE Healthcare) with the following acquisition parameters: 120 $\mathrm{kV}$ (peak), 250-330 mAs, 0.531 pitch, 1.0-second rotation time. Multiplanar reconstructions were generated, including 0.625- $\mathrm{mm}$
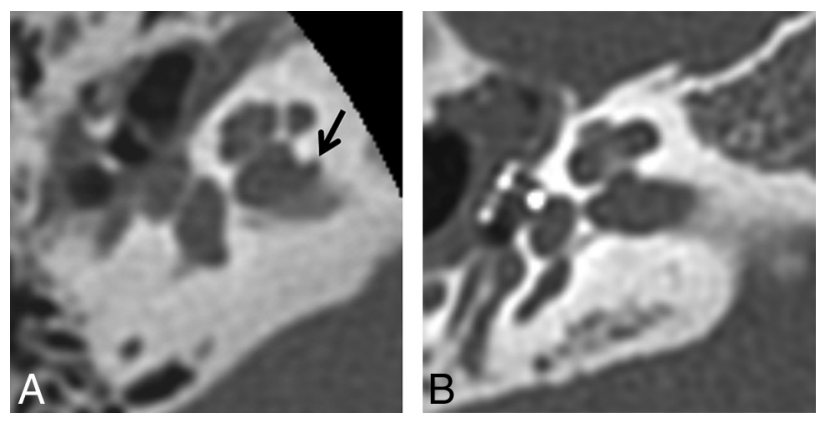

FIG 1. Axial temporal bone CT image $(A)$ at the level of the inferior aspect of the right internal auditory canal demonstrates the typical appearance of an internal auditory canal diverticulum (arrow). An image obtained at the same level in a different patient without an internal auditory canal diverticulum is provided for comparison $(B)$.

axial (9.6-cm FOV; bone plus kernel) and 0.6-mm coronal (9.6cm FOV; bone plus kernel) images.

\section{Reader Assessment}

Four fellowship-trained neuroradiologists (3 years', 12 years', 4 years', and 6 years' subspecialty experience) served as readers for this retrospective study. Each reader participated in an initial image review as well as a subsequent image review. For both image reviews, the neuroradiologists were instructed to first assess the axial $0.625 \mathrm{~mm}$ bone algorithm images to determine the presence or absence of an IAC diverticulum (Fig 1). If necessary or desired, the coronal bone algorithm images could also be used to aid in making this determination. A subjective determination of the presence or absence of an IAC diverticulum was used rather than a minimum size threshold to be consistent with the described methods of Mihal et al, ${ }^{1}$ Puac et al, ${ }^{5}$ and Pippin et al. ${ }^{8}$ In addition to the initial and subsequent image reviews in which the 4 neuroradiologists participated, 1 of the neuroradiologists (3 years' subspecialty experience) reviewed the temporal bone CT images to determine the presence or absence of an enlarged vestibular aqueduct, labyrinthine dysplasia, cochlear cleft, and otospongiosis.

Initial Review. For the initial image review, the readers were instructed to assess the temporal bone CT images for the presence or absence of IAC diverticula based on their current understanding of this entity as described in the literature. This nonstandardized initial review was intended to facilitate assessment of interobserver variability in the identification of IAC diverticula among practicing neuroradiologists. For the initial review, 100 temporal bone CT examinations were evaluated by all 4 neuroradiologists, and interrater reliability was calculated on the basis of the recorded assessments of these 100 examinations. All remaining temporal bone CT examinations were assessed by 2 neuroradiologist readers.

Subsequent Review. After the 4 neuroradiologists had completed their initial reviews, the group discussed the observed variability of the results and reviewed together a total of 10 representative discrepant cases selected from the 100-case cohort reviewed by all 4 neuroradiologists. On the basis of this discussion and 

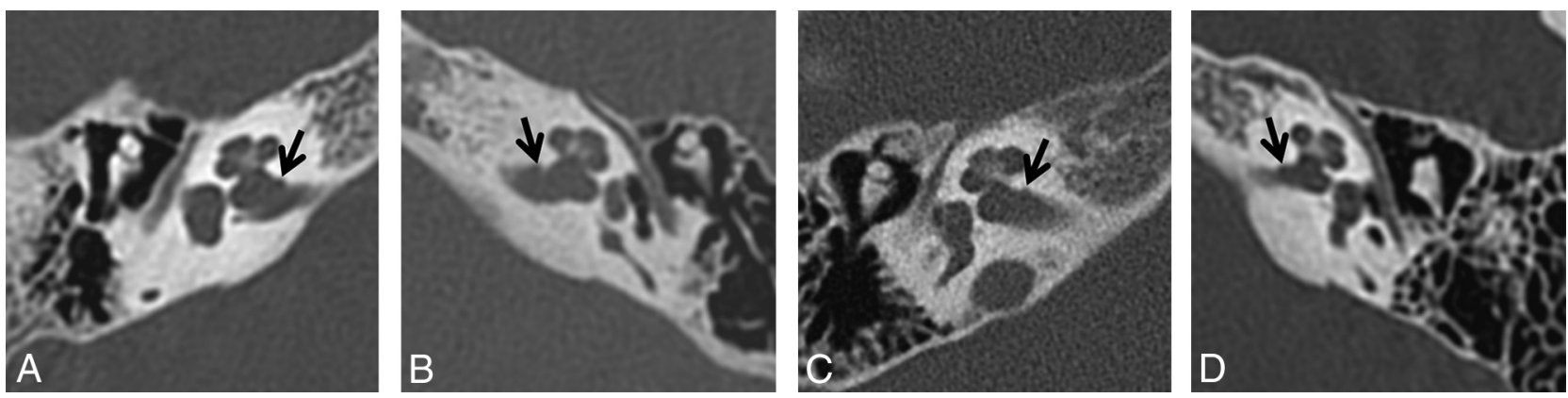

FIG 2. Visual threshold developed after the initial review and applied during the subsequent review by the 4 neuroradiologist readers. The visual threshold consists of axial temporal bone $\mathrm{CT}$ images obtained in 4 different patients and is intended to depict the minimum contour irregularity necessary to be considered an internal auditory canal diverticulum (arrows) for the second review performed in this study.

\section{Table 1: Characteristics of the study group}

\begin{tabular}{lc}
\hline Sex & \\
Male & $157(55 \%)$ \\
Female & $126(45 \%)$ \\
Age & \\
Mean (SD) (yr) & $7.8(4.8)$ \\
Minimum & 6 weeks \\
Maximum & 17 years \\
Audiogram (temporal bone sides) & \\
Normal & $152(27 \%)$ \\
Sensorineural loss & $158(28 \%)$ \\
Mixed loss & $39(7 \%)$ \\
Conductive loss & $112(20 \%)$ \\
Unavailable & $100(18 \%)$ \\
\hline
\end{tabular}

Table 2: Temporal bone CT findings within the study group

\begin{tabular}{lc}
\hline \multicolumn{2}{l}{ Individual Temporal Bones (Sides) } \\
\hline Internal auditory canal diverticulum & $64(11 \%)$ \\
Yes & $497(89 \%)$ \\
No & \\
Enlarged vestibular aqueduct & $43(8 \%)$ \\
Yes & $518(92 \%)$ \\
No & \\
Labyrinthine dysplasia & $66(12 \%)$ \\
Yes & $495(88 \%)$ \\
No & \\
Cochlear cleft & $205(37 \%)$ \\
Yes & $356(63 \%)$ \\
No & \\
Otospongiosis & $2^{\text {a }}(0.4 \%)$ \\
Yes & $559(99.6 \%)$ \\
No &
\end{tabular}

discrepant case review, a consensus visual threshold for what should be called an IAC diverticulum (Fig 2) was proposed and developed for use in the second review, with the understanding that a candidate diverticulum should be at least as conspicuous as the examples in the agreed-upon threshold to be considered present.

All temporal bone CT examinations identified by at least 1 neuroradiologist as showing an IAC diverticulum (unilateral or bilateral) in the initial review were included in the second review to be reassessed. The examinations for which no neuroradiologist identified an IAC diverticulum on the initial review were considered not to contain an IAC diverticulum and were, therefore, excluded from the second review. The time interval between the initial and subsequent reviews was 2 months.

For the subsequent image review, all 4 neuroradiologists reviewed the CT images of the included temporal bone CT examinations (both sides) to assess the presence of an IAC diverticulum using the agreed-upon visual threshold. The recorded results were reviewed, and discrepancies were resolved by consensus.

\section{Statistical Analysis}

Mean, SD, and range are reported for continuous variables. Absolute and relative frequencies are reported for categoric variables. The Fisher exact test was used to compare proportions. One-way analysis of variance was used to compare continuous variables. The Light $\kappa$ coefficient was calculated to assess interrater reliability. All analyses were performed with JMP, Version 14 (SAS Institute), and a $P$ value $<.05$ indicated a statistically significant difference.

\section{RESULTS}

\section{Subjects}

A total of 284 temporal bone CT examinations (568 sides) were reviewed. Among these, 6 sides were excluded for artifacts precluding diagnostic assessment of the IAC, and 1 side was excluded for congenital absence of the IAC, which yielded a study cohort of 283 patients and 561 temporal bones. Audiologic data were available in 234 patients (82.6\%). One patient $(0.4 \%)$ had a clinical diagnosis of otospongiosis. Patient age, sex, and audiogram data are summarized in Table 1. Temporal bone CT findings are summarized in Table 2.

\section{Reader Assessment}

Initial Review. On the initial review of 283 patients (561 individual temporal bones), neuroradiologist 1 identified IAC diverticula in $19.1 \%$ of individual temporal bones ( $24.7 \%$ of patients), neuroradiologist 2 identified IAC diverticula in $13.1 \%$ of temporal bones ( $8.5 \%$ of patients), neuroradiologist 3 identified IAC diverticula in $9.0 \%$ of temporal bones (9.2\% of patients), and neuroradiologist 4 identified IAC diverticula in $5.1 \%$ of temporal bones (3.5\% of patients). For the 100 examinations reviewed by all 4 


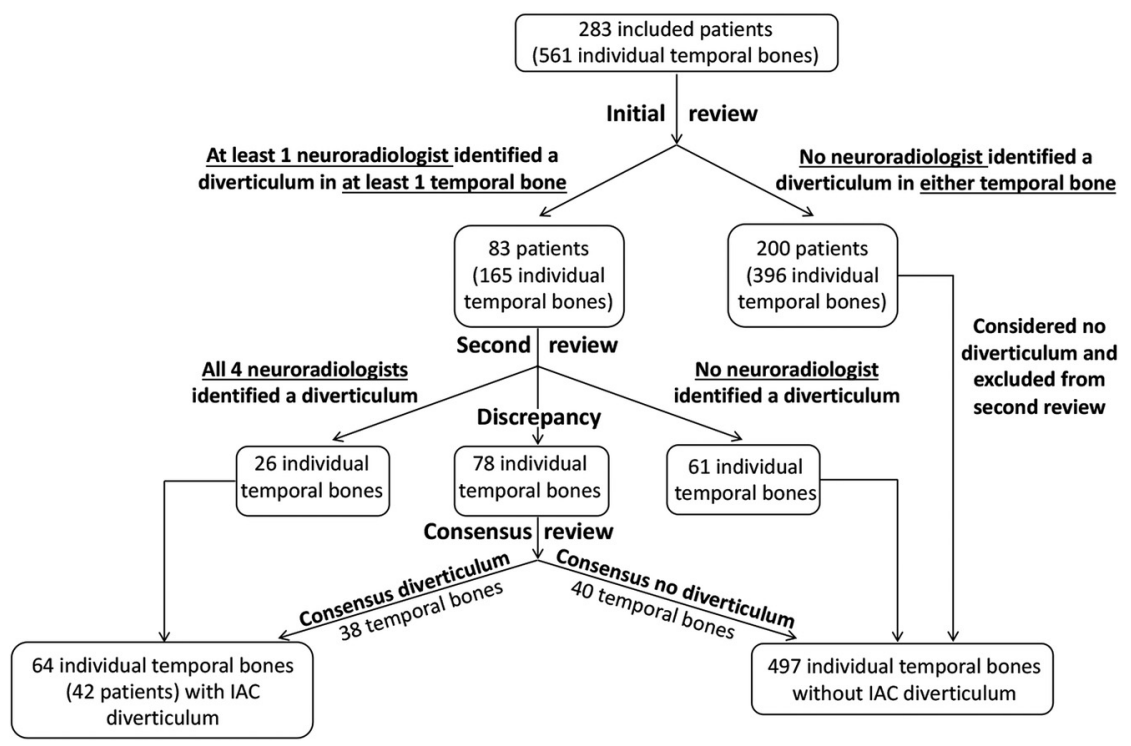

FIG 3. Flow chart depicting the initial and subsequent image reviews.

Table 3: Internal auditory canal diverticula association assessment

\begin{tabular}{|c|c|c|c|}
\hline & \multicolumn{2}{|c|}{ Internal Auditory Canal Diverticulum } & \multirow[b]{2}{*}{$P$} \\
\hline & Yes & No & \\
\hline \multicolumn{4}{|l|}{$\operatorname{Sex}^{a}$} \\
\hline Male & 28 & 129 & .13 \\
\hline Female & 14 & 112 & \\
\hline Age (mean) (SD) (yr) & $7.7(5.0)$ & $7.8(4.8)$ & .91 \\
\hline \multicolumn{4}{|l|}{ Audiogramb } \\
\hline Normal hearing & 20 & 132 & .65 \\
\hline Hearing loss & 36 & 273 & \\
\hline Conductive & 16 & 96 & .86 \\
\hline Sensorineural & 16 & 142 & .48 \\
\hline Mixed & 4 & 35 & .79 \\
\hline \multicolumn{4}{|c|}{ Enlarged vestibular aqueduct ${ }^{\mathrm{b}}$} \\
\hline Yes & 2 & 41 & .21 \\
\hline No & 62 & 456 & \\
\hline \multicolumn{4}{|c|}{ Labyrinthine dysplasia $^{\mathrm{b}}$} \\
\hline Yes & 7 & 59 & 1.00 \\
\hline No & 57 & 438 & \\
\hline \multicolumn{4}{|l|}{ Cochlear cleft ${ }^{\mathrm{b}}$} \\
\hline Yes & 30 & 175 & .07 \\
\hline No & 34 & 322 & \\
\hline \multicolumn{4}{|l|}{ Otospongiosis ${ }^{\mathrm{b}}$} \\
\hline Yes & 2 & 0 & .013 \\
\hline No & 62 & 497 & \\
\hline
\end{tabular}

${ }^{\mathrm{a}}$ Denotes number of patients.

${ }^{\mathrm{b}}$ Denotes number of individual temporal bones (sides).

neuroradiologists, the Light $\kappa$ coefficient was 0.44 , indicating moderate agreement.

Subsequent Review. A total of 83 temporal bone CT examinations were included in the subsequent review (Fig 3), having been identified by at least 1 neuroradiologist as having an IAC diverticulum on at least 1 side. Following consensus review, IAC diverticula were present in 42 patients ( $14.8 \%$ of the 283 subject cohort) (mean age, $7.7 \pm 5.0$ years; range, 2 months to 17 years) and 64 IACs (11.4\% of the 561 included individual temporal bones). IAC diverticula were bilateral in 22 patients $(52.4 \%)$ and unilateral in 20 patients (47.6\%). For these 83 temporal bone CT examinations included in the subsequent review, the Light $\kappa$ coefficient was 0.48 , indicating moderate agreement.

\section{Association Assessment}

There was no statistically significant association between IAC diverticula (Table 3$)$ and age $(P=.91)$. IAC diverticula were more common among males $(17.8 \%)$ than females $(11.0 \%)$; however, this observed difference was not statistically significant $(P=.13)$. No statistically significant association was observed between IAC diverticula and conductive $(P=.86)$, sensorineural $(P=.48)$, or mixed hearing loss $(P=.79)$. Additionally, there were no statistically significant associations between IAC diverticula and an enlarged vestibular aqueduct $(P=.21)$, labyrinthine dysplasia $(P=.1 .00)$, or cochlear cleft $(P=.07)$. The 1 child in the study cohort with otospongiosis also had bilateral IAC diverticula; thus, a statistically significant association between IAC diverticula and otospongiosis $(P=.013)$ was observed.

\section{DISCUSSION}

IAC diverticula have been a topic of interest in the recent radiologic ${ }^{1,5,8}$ and otolaryngologic $2,9,11,12$ literature, and there is uncertainty with respect to the underlying etiology and clinical relevance of this finding. IAC diverticula have been described in otospongiotic temporal bone specimens, ${ }^{13,14}$ have been identified in $>30 \%$ of patients with otospongiosis, ${ }^{5,12}$ and may be associated with poorer postoperative audiologic outcomes following stapedotomy for otospongiosis. ${ }^{11}$ However, IAC diverticula have also been identified histologically ${ }^{2,6,7}$ and radiologically $^{1,8,9}$ in the absence of clinical, histopathologic, or imaging evidence of otospongiosis. Although there are some data suggesting an association between isolated IAC diverticula and sensorineural hearing loss, ${ }^{8}$ the preponderance of evidence indicates that isolated IAC diverticula are more likely a normal anatomic variant. $^{1,2,9}$

The current study documents that isolated IAC diverticula, occurring in the absence of otospongiosis, are a relatively common finding on pediatric temporal bone CT, occurring in nearly $15 \%$ of patients and comprising $97 \%$ of IAC diverticula in this 

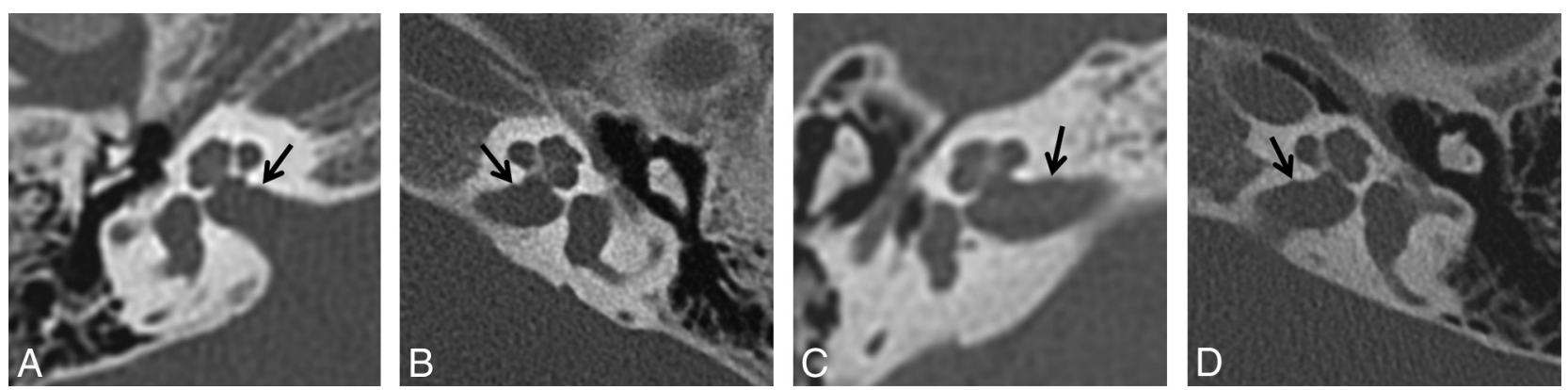

FIG 4. Axial temporal bone CT images from 4 different patients depict representative examples of subtle IAC contour irregularities (arrows) determined during consensus review of discrepant cases to not reach the visual threshold necessary to be considered an IAC diverticulum.

cohort. Furthermore, this study supports the hypothesis that isolated IAC diverticula fall within the range of normal anatomic variation because no statistically significant associations were observed between IAC diverticula and hearing loss, enlarged vestibular aqueduct, or labyrinthine dysplasia.

Only 1 patient in this pediatric cohort had clinical or imaging evidence of otospongiosis; however, this 1 patient also had bilateral IAC diverticula. Although firm conclusions should not be drawn on the basis of findings in a single patient, this association is in keeping with previous work indicating that IAC diverticula are more frequently observed in the presence of otospongiosis ${ }^{5,12}$ and hypothesizing a predilection for otospongiosis to involve the anterior-inferior aspect of the IAC fundus in addition to the region of the fissula ante fenestram. ${ }^{5,8}$

The observed prevalence of IAC diverticula in this pediatric cohort ( $11.4 \%$ of IACs, $14.8 \%$ of patients) is higher than the $5 \%-$ $6 \%$ prevalence reported in previous large radiologic studies ${ }^{1,8,9}$ but less than the $25 \%$ prevalence reported in a small histologic study. ${ }^{2}$ The reasons for the higher observed prevalence in this study compared with previous radiologic studies are unclear and warrant further investigation.

One possibility is that the consensus visual threshold used in this study represents a lower threshold for classifying an IAC diverticulum as present relative to previous published work on the topic. However, this is difficult to determine with confidence because a similar visual threshold was not provided with other studies of IAC diverticula. ${ }^{1,5,8}$ Of note, Mihal et $\mathrm{al}^{1}$ reported that $25 \%$ of isolated IAC diverticula observed in their study were $<0.9 \mathrm{~mm}$, and Pippin et $\mathrm{al}^{8}$ described the appearance of some IAC diverticula as "a small notch."

Another possible explanation for the higher prevalence of IAC diverticula observed in this pediatric study compared with previous radiologic studies performed predominantly or exclusively in adults is that IAC diverticula represent an embryologic remnant of the formation of the otic capsule from 14 ossification centers analogous to the fissula ante fenestram, the fossula post fenestram, and the hypoattenuated focus in the anterior otic capsule., ${ }^{2,15-17}$ In such a scenario, the higher prevalence of IAC diverticula in this pediatric cohort relative to larger adult cohorts could be explained if in most children, the incompletely mineralized bone or cartilage corresponding to IAC diverticula became replaced or filled in by fully mineralized bone over the course of development, as is believed to occur with the cochlear cleft ${ }^{18}$ and the hypoattenuated focus in the anterior otic capsule. ${ }^{17}$ An association between IAC diverticula and the cochlear cleft as well as younger age might be expected if this hypothesis were true; however, none was observed in this study. These possibilities could be further investigated by a longitudinal study.

A secondary goal of this study was to assess interobserver variability in the identification of IAC diverticula, which has not been a focus of previous reports. In both our initial and subsequent reviews, there was substantial interobserver variability among the 4 experienced neuroradiologist readers in determining whether an IAC diverticulum was present despite the implementation of an agreed-upon visual threshold. During consensus review, discussions often centered on whether a subtle contour irregularity simply represented mild generalized undulation of the IAC versus a focal IAC diverticulum (Fig 4). These findings suggest that other neuroradiologists may also face uncertainty as to what exactly does and does not constitute an IAC diverticulum on temporal bone CT for purposes of both clinical practice and future research. Although our findings suggest isolated IAC diverticula to be a normal anatomic variant without clinical significance, a clear, easily applicable IAC diverticulum definition or threshold with relatively low interobserver variability would be useful for future research studies and particularly important in the event that isolated IAC diverticula are someday shown to be clinically relevant.

\section{CONCLUSIONS}

Internal auditory canal diverticula are a common finding on pediatric temporal bone CT, seen in approximately $15 \%$ of patients and $11 \%$ of IACs. There is no statistically significant association with hearing loss or congenital inner ear anomaly. In the absence of clinical or imaging evidence of otospongiosis, internal auditory canal diverticula likely fall within the range of normal anatomic variation. Familiarity with these findings may prevent neuroradiologists from recommending unnecessary additional testing in pediatric patients with isolated internal auditory canal diverticula.

\section{REFERENCES}

1. Mihal DC, Feng Y, Kodet ML, et al. Isolated internal auditory canal diverticula: a normal anatomic variant not associated with sensorineural hearing loss. AJNR Am J Neuroradiol 2018;39:234044 CrossRef Medline

2. Muelleman T, Maxwell AK, Lopez I, et al. Histopathologic characteristics of internal auditory canal diverticula. Otol Neurotol 2019;40:e653-56 CrossRef Medline 
3. Makarem AO, Hoang TA, Lo WW, et al. Cavitating otosclerosis: clinical, radiologic, and histopathologic correlations. Otol Neurotol 2010;31:381-84 CrossRef Medline

4. Bou-Assaly W, Mukherji S, Srinivasan A. Bilateral cavitary otosclerosis: a rare presentation of otosclerosis and cause of hearing loss. Clin Imaging 2013;37:1116-18 CrossRef Medline

5. Puac P, Rodríguez A, Lin HC, et al. Cavitary plaques in otospongiosis: CT findings and clinical implications. AJNR Am J Neuroradiol 2018;39:1135-39 CrossRef Medline

6. Kollias S. Imaging of the congenitally malformed temporal bone. In: Lemmerling M, Kollias S, eds. Radiology of the Petrous Bone. SpringerVerlag; 2004:121

7. Gulya A, Schuknecht H. The inner ear. In: Gulya A, ed. Gulya and Schuknecht's Anatomy of the Temporal Bone with Surgical Implications. 3rd ed. Informa Healthcare; 2007:168-69

8. Pippin KJ, Muelleman TJ, Hill J, et al. Prevalence of internal auditory canal diverticulum and its association with hearing loss and otosclerosis. AJNR Am J Neuroradiol 2017;38:2167-71 CrossRef Medline

9. Muelleman TJ, Pippin K, Shew M, et al. The size of internal auditory canal diverticula is unrelated to degree of hearing loss. Laryngoscope 2020;130:1011-15 CrossRef Medline

10. Lescanne E, Bakhos D, Metais JP, et al. Otosclerosis in children and adolescents: a clinical and CT-scan survey with review of the literature. Int J Pediatr Otorhinolaryngol 2008;72:147-52 CrossRef Medline

11. Shim YJ, Bae YJ, An GS, et al. Involvement of the internal auditory canal in subjects with cochlear otosclerosis: a less acknowledged third window that affects surgical outcome. Otol Neurotol 2019;40: e186-90 CrossRef Medline

12. Wang F, Yoshida T, Shimono M, et al. Significance of internal auditory canal diverticula in ears with otosclerosis. Acta Otolaryngol 2018;138:1066-69 CrossRef Medline

13. Schuknecht HF, Barber W. Histologic variants in otosclerosis. Laryngoscope 1985;95:1307-17 CrossRef Medline

14. Hueb MM, Goycoolea MV, Paparella MM, et al. Otosclerosis: the University of Minnesota temporal bone collection. Otolaryngol Head Neck Surg 1991;105:396-405 CrossRef Medline

15. Anson BJ, Cauldwell EW, Bast TH. The fissula ante fenestram of the human otic capsule; developmental and normal adult structure. Ann Otol Rhinol Laryngol 1947;56:957-85 CrossRef Medline

16. Bast T, Anson B. The Temporal Bone and the Ear. CC Thomas; 1949

17. Moser T, Veillon F, Sick H, et al. The hypodense focus in the petrous apex: a potential pitfall on multidetector CT imaging of the temporal bone. AJNR Am J Neuroradiol 2008;29:35-39 CrossRef Medline

18. Chadwell JB, Halsted MJ, Choo DI, et al. The cochlear cleft. AJNR Am J Neuroradiol 2004;25:21-24 Medline 